\section{DYNAMICAL LOAD OF LINEAR ROLLING GUIDES}

RADKA JIROVA, LUBOMIR PESIK

Technical University of Liberec, Liberec, Czech Republic

DOI : 10.17973/MMSJ.2020_06_2020014

radka.jirova@tul.cz

Linear rolling guides are used for realizing a translational motion of mechanical elements or assemblies e.g. in industrial robots, handling or CNC machines. Their reliability is highly desirable to meet the requirements of Industry 4.0, and hence the accurate load identification of linear guides is needed. The objective of this article is to identify a dynamical load of linear guides based on the substitutive mathematical model of machines. Where, the kinematic excitation, mass, inertia parameters of moving bodies and elastic and damping behaviour of linear guides are defined. The simulation of specific operating conditions is reached by Lagrange equations in a vector form. This way conveniently enables respecting the effect of nonconservative forces in the calculation. The article comprehensively describes the method of the dynamical load calculation of linear rolling guides, which is usable for a wide range of machines, their design as well as the design of linear guides.

KEYWORDS

dynamical load, linear rolling guides, Lagrange equations, dynamical analysis, multibody systems

\section{INTRODUCTION}

Nowadays, for minimization of production losses and to meet requirements of Industry 4.0, the high reliability of linear rolling guides is demanded. These are widely used in machines of production lines, where enable linear motion of machine elements or assemblies. The most common usage examples are industrial robots, handling and CNC machines.

Efforts to increase the reliability are often reflected in the detailed description of linear guides wear that is directly related to the contact stress of contact surfaces [Li 2018], [Kwon 2019]. For example, [Wei 2017b] evaluates the effect of the linear guides wear on the contact stiffness change. Another principle for the evaluation of the contact stresses uses [Ohta 2019] and [Cheng 2018], who determine the influence of the friction on the contact stress increase by FEM analysis and by analytic calculation. [Shimizu 2008] examines the influence of contact stresses on the basic dynamical capacity of linear guides.

The above described knowledge of the fatigue wear is further accounted into the damage diagnostics of linear rolling guides [THK 2011], [THK 2019]. For example, [Schaeffler Technologies 2017] for determination of linear guides damage uses time sections that respond to the vibration energy increase under the relubrication of linear guides. [Feng 2018] uses the analogous model by applying the wavelet transform and the neural networks for the signal analysis. [Wei 2017a] describes the reliability analysis of linear guides dynamically loaded and [Tao 2013] proposes the damage prediction based on the predetermined model of linear guides wear under a variable load and trajectory conditions.

Nevertheless, linear rolling guides are currently designed mainly according to the static part of the load, but the significant portion of the linear guide's load is a dynamical part of that load caused by the inertia forces of the moving bodies. Therefore, the dynamical part of the load needs to be added to the first draft of machines with linear guides as well as to the investigation of contact stresses or diagnostic methods.

Currently, many conventional engineering tools can be used for computing the bodies' motion and evaluating the dynamical forces. These multibody system analyses (MBS) mainly use augmented Lagrange equations [Wittenburg 2008], [Schiehlen 2007]. In the case of machines with linear guides, using Lagrange equations with a member of constraint is complicated especially for the evaluating the dynamical forces to the linear guide that can be represented by a series of elastic and damping links [Ohta 2000]. Further complication also might be creating the proper kinematic pairs those constrain the basic motion of the solved system.

Hence, the article is focused on the calculation of the linear guides' dynamical load using the mathematical model based on Lagrange equations in a vector form. The load calculation is further shown in a practical example.

\section{DESIGN AND USE OF LINEAR GUIDES}

The linear guide consists of the carriage, where rolling elements are situated and those are in contact with a guiding profile. A moving body of the machine is usually connected to the carriage; the guiding profile is connected to the machine frame (Fig. 1).

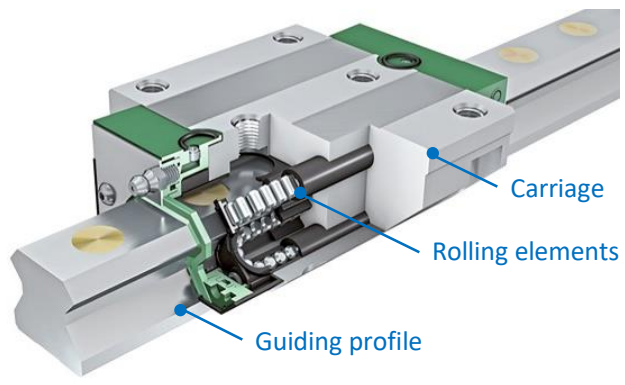

Figure 1. The linear rolling guide [Schaeffler Group 2020]

An example of linear guides' usage, a manipulator of glass panes, is shown in Fig. 2. The moving assembly of this machine is composed of a cart with a telescopic carrier of clamping frames. In the manipulator, linear guide carriages are used on two parallel guiding profiles. The configuration of the moveable assembly and kinematic conditions are cyclically changed during the manipulator operation; mass and inertia parameters are consequently changed too. Various static and dynamical loads appertain to each assembly configuration and kinematic conditions

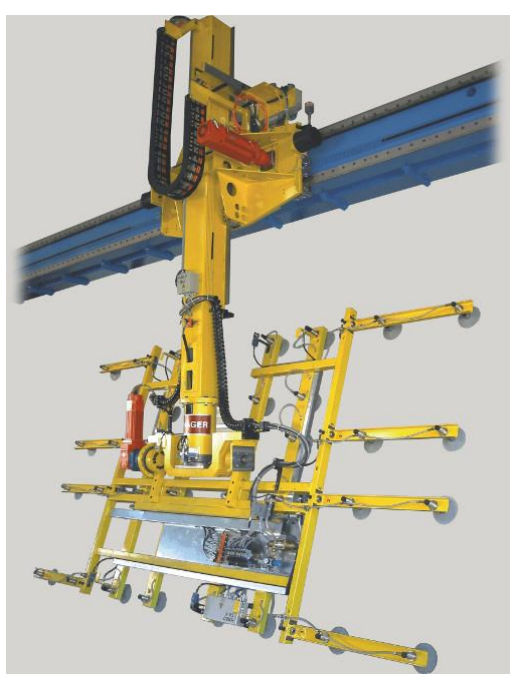

Figure 2. The glass pane manipulator [HAGER 2020] 


\section{LAGRANGE EQUATIONS}

The Lagrange equations in the vector form are used for the load evaluation of linear guides. Their advantage is the machine system description based on energies and on a proper set of generalized coordinates and generalized forces relate to the generalized coordinates. In this case, considering the dynamical behaviour of solved systems, the advantage is usage of Lagrange equations without a member of constraint. The proposed computation is mainly advantageous for easier optimization of dynamical parameters, better control of computing steps and settings, and especially for a more clear evaluation of the dynamical load. The chapter describes the derivation of the Lagrange equation in the vector form up to the final differential equations of motion. This calculation is applicable for diverse one mass systems with a spatial and translational motion via linear guides. It should be noted that one mass system is suitable for machines, where the dynamical load of linear guides is not much influenced by the dynamics of structural mechanisms or gears.

The load calculation of linear guides substitutes solved systems by the mathematical model. The mathematical model is represented by a one mass system with a kinematic excitation defined as a time acceleration function at the reference point.

The dynamical system (Fig. 3 ) is defined by mass and inertia parameters of a virtual body of the solved machine. Linear guides are substituted by elastic and damping links of the virtual body against the frame. Their stiffness and damping coefficient matches the parameters given by producers.

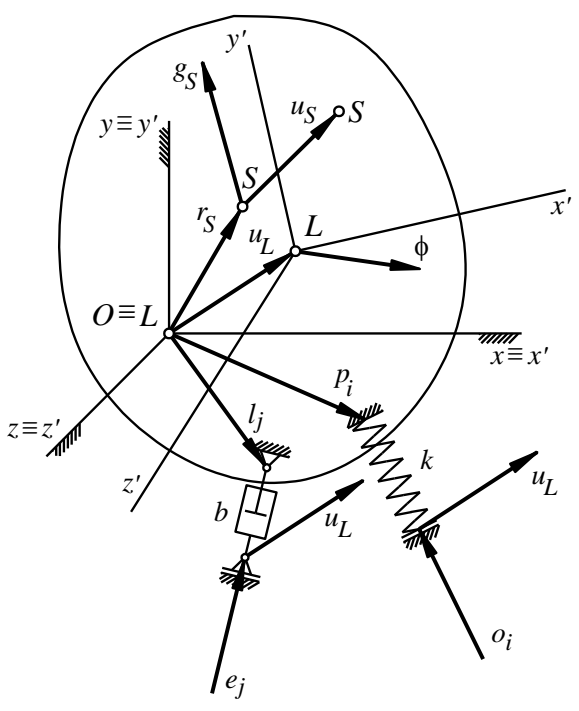

Figure 3. The one mass system

The motion of the virtual body is described in global coordinates $x, y, z$ with the origin $O$. Simultaneously, at time $t_{0}$ is the local coordinate system of the virtual body $x^{\prime}, y^{\prime}, z^{\prime}$ with the origin $L$ identical to the global coordinate system. At time $t$, the origin of the local coordinate system is shifted by the position vector $\boldsymbol{u}_{\boldsymbol{L}}$ and rotated by the angular position vector $\boldsymbol{\varphi}$. The proper set of generalized coordinates respectively generalized velocities is represented by the position vector $\boldsymbol{u}_{\boldsymbol{L}}$ and the angular position vector $\boldsymbol{\varphi}$ respectively the velocity vector $\boldsymbol{u}_{\mathbf{L}}^{\prime}$ and the angular velocity vector $\boldsymbol{\varphi}^{\prime}$. The Lagrange equation of spatial motion in the vector form is

$$
\frac{d}{d t}\left(\frac{\partial K}{\partial \boldsymbol{q}^{\prime}}\right)-\frac{\partial K}{\partial \boldsymbol{q}}+\frac{\partial U}{\partial \boldsymbol{q}}+\frac{\partial D}{\partial \boldsymbol{q}^{\prime}}=\boldsymbol{Q}
$$

wherein $K$ is kinetic energy, $U$ potential energy, $D$ dissipative energy, $\boldsymbol{Q}$ generalized force and moment, $\boldsymbol{q}$ generalized coordinate and $\boldsymbol{q}^{\prime}$ generalized velocity.

The proposed solution defines the kinetic energy $K$ of the virtual body as a scalar field, which is given by the square of the scalar multiplication of the velocity vector. Analogously, the dissipative energy $D$ of damping links and the potential energy $U$ of elastic links is defined.

\subsection{Kinetic energy}

The kinetic energy of the rigid body is

$$
K=\frac{1}{2} \int_{m} \boldsymbol{u}^{\prime 2} d m
$$

The velocity vector $\boldsymbol{u}^{\prime}$ is defined

$$
u^{\prime}=u_{L}^{\prime}+\varphi^{\prime} \times r
$$

Whenever the antisymmetric tensor $\boldsymbol{R}$ of the vector $\boldsymbol{r}$ exists, the equation (3) becomes

$$
\boldsymbol{u}^{\prime}=\boldsymbol{u}_{L}^{\prime}-\boldsymbol{R} \varphi^{\prime}
$$

wherein the antisymmetric tensor $\boldsymbol{R}$ is

$$
\boldsymbol{R}=\left[\begin{array}{ccc}
0 & -z & y \\
z & 0 & -x \\
-y & x & 0
\end{array}\right]
$$

The partial derivative of the kinetic energy $K$ by the velocity vector $\boldsymbol{u}_{\boldsymbol{L}}{ }^{\prime}$ is then

$$
\begin{aligned}
& \frac{\partial K}{\partial \boldsymbol{u}_{\boldsymbol{L}}^{\prime}}= \\
& =\int_{m}\left(\boldsymbol{u}_{\boldsymbol{L}}^{\prime}-\boldsymbol{R} \boldsymbol{\varphi}^{\prime}\right) \operatorname{Grad}_{\boldsymbol{u}_{\boldsymbol{L}}^{\prime}}\left(\boldsymbol{u}_{\boldsymbol{L}}^{\prime}-\boldsymbol{R} \boldsymbol{\varphi}^{\prime}\right) d m= \\
& =\int_{m}\left(\boldsymbol{u}_{\boldsymbol{L}}^{\prime}-\boldsymbol{R} \boldsymbol{\varphi}^{\prime}\right) \boldsymbol{I} d m=m \boldsymbol{u}_{\boldsymbol{L}}^{\prime}-\boldsymbol{S}_{\boldsymbol{L}} \boldsymbol{\varphi}^{\prime}
\end{aligned}
$$

wherein $\boldsymbol{I}$ is the unit tensor.

$$
\boldsymbol{I}=\left[\begin{array}{lll}
1 & 0 & 0 \\
0 & 1 & 0 \\
0 & 0 & 1
\end{array}\right]
$$


and $S_{L}$ is the antisymmetric tensor related to the vector of the first moment of inertia respecting the origin $L$ of the local coordinate system

$$
\boldsymbol{S}_{\boldsymbol{L}}=\left[\begin{array}{ccc}
0 & -S_{x y} & S_{x z} \\
S_{x y} & 0 & -S_{y z} \\
-S_{x z} & S_{y z} & 0
\end{array}\right]
$$

The partial derivative of the kinetic energy $K$ by the angular velocity vector $\boldsymbol{\varphi}^{\prime}$ is

$$
\begin{aligned}
& \frac{\partial K}{\partial \boldsymbol{\varphi}^{\prime}}= \\
& =\int_{m}\left(\boldsymbol{u}_{\boldsymbol{L}}^{\prime}-\boldsymbol{R} \boldsymbol{\varphi}^{\prime}\right) \operatorname{Grad}_{\boldsymbol{\varphi}^{\prime}}\left(\boldsymbol{u}_{\boldsymbol{L}}^{\prime}-\boldsymbol{R} \boldsymbol{\varphi}^{\prime}\right) d m= \\
& =\int_{m}\left(\boldsymbol{u}_{\boldsymbol{L}}^{\prime}-\boldsymbol{R} \boldsymbol{\varphi}^{\prime}\right)(-\boldsymbol{R}) d m= \\
& =\int_{m}\left(\boldsymbol{R} \boldsymbol{u}_{\boldsymbol{L}}^{\prime}-\boldsymbol{R}^{2} \boldsymbol{\varphi}^{\prime}\right) d m=\boldsymbol{S}_{\boldsymbol{L}} \boldsymbol{u}_{\boldsymbol{L}}^{\prime}+\boldsymbol{J}_{\boldsymbol{L}} \boldsymbol{\varphi}^{\prime}
\end{aligned}
$$

wherein $\boldsymbol{J}_{\mathbf{L}}$ is tensor of inertia respecting the origin $L$ of the local coordinate system.

$$
\boldsymbol{J}_{\boldsymbol{L}}=\left[\begin{array}{ccc}
J_{L x} & -D_{L x y} & -D_{L x z} \\
-D_{L x y} & J_{L y} & -D_{L y z} \\
-D_{L x z} & -D_{L y z} & J_{L z}
\end{array}\right]
$$

The first member of the equation (1) for the generalized coordinates $\boldsymbol{q}^{\prime}$, thus for the velocity vector $\boldsymbol{u}_{\mathbf{L}}{ }^{\prime}$ and the angular velocity vector $\boldsymbol{\varphi}^{\prime}$ becomes

$$
\begin{aligned}
& \frac{d}{d t}\left(\frac{\partial K}{\partial \boldsymbol{u}_{L}^{\prime}}\right)=m \boldsymbol{u}^{\prime \prime}{ }_{L}-\boldsymbol{S}_{\boldsymbol{L}} \boldsymbol{\varphi}^{\prime \prime} \\
& \frac{d}{d t}\left(\frac{\partial K}{\partial \boldsymbol{\varphi}^{\prime}}\right)=\boldsymbol{S}_{\boldsymbol{L}} \boldsymbol{u}^{\prime \prime}{ }_{\boldsymbol{L}}+\boldsymbol{J}_{\boldsymbol{L}} \boldsymbol{\varphi}^{\prime \prime}
\end{aligned}
$$

\subsection{Potential energy}

The energy of elastic links against the equilibrium position and by considering the gravitational field, the potential energy $U$ becomes

$$
U=\sum_{i=1}^{n} \frac{1}{2} k_{i} \delta_{i}^{2}+m g \boldsymbol{u}_{\boldsymbol{S}} \boldsymbol{g}_{\boldsymbol{S}}
$$

wherein $k_{i}$ is the stiffness of $i$-th elastic link and $\delta_{i}$ its deformation, $m$ is mass of the body, $g$ the gravitational acceleration, $\boldsymbol{u}_{\boldsymbol{s}}$ the position vector of the centre of mass and $\boldsymbol{g}_{s}$ the unit vector in the gravity direction.
The deformation $\delta_{i}$ of the elastic link may be expressed as the scalar multiplication of the relative position $\Delta \boldsymbol{u}_{i}$ of its endpoint against the start point and the unit vector $\boldsymbol{o}_{\boldsymbol{i}}$ in the elastic link direction. Wherein the endpoint position $\boldsymbol{u}_{\boldsymbol{i}}$ of $\boldsymbol{i}$-th elastic link is

$$
u_{i}=u_{L}+\varphi \times p_{i}
$$

and $\boldsymbol{u}_{L i}$ is the start point position of $i$-th elastic link. The deformation $\delta_{i}$ is then

$$
\delta_{i}=\Delta \boldsymbol{u}_{i} \boldsymbol{o}_{i}=\left(\boldsymbol{u}_{i}-\boldsymbol{u}_{L i}\right) \boldsymbol{o}_{i}
$$

The position vector $\boldsymbol{u}_{\boldsymbol{s}}$ is defined

$$
u_{S}=u_{L}+\varphi \times r_{S}
$$

Equations (14) and (16), analogously to the equation (3) and (4), become

$$
u_{i}=u_{L}-P_{i} \varphi
$$

and

$$
u_{S}=u_{L}-R_{S} \varphi
$$

wherein $\boldsymbol{P}_{\boldsymbol{i}}$ is antisymmetric tensor related to the vector $\boldsymbol{p}_{\boldsymbol{i}}$

$$
\boldsymbol{P}_{\boldsymbol{i}}=\left[\begin{array}{ccc}
0 & -p_{z i} & p_{y i} \\
p_{z i} & 0 & -p_{x i} \\
-p_{y i} & p_{x i} & 0
\end{array}\right]
$$

and $\boldsymbol{R}_{\boldsymbol{S}}$ is antisymmetric tensor related to the vector $\boldsymbol{r}_{\boldsymbol{s}}$.

$$
\boldsymbol{R}_{\boldsymbol{S}}=\left[\begin{array}{ccc}
0 & -r_{S z} & r_{S y} \\
r_{S z} & 0 & -r_{S x} \\
-r_{S y} & r_{S x} & 0
\end{array}\right]
$$

The partial derivative of the potential energy $U$ by the position vector $\boldsymbol{u}_{\boldsymbol{L}}$ is

$$
\begin{aligned}
& \frac{\partial U}{\partial \boldsymbol{u}_{\boldsymbol{L}}}=\sum_{i=1}^{n} k_{i}\left(\boldsymbol{u}_{\boldsymbol{L}}-\boldsymbol{P}_{\boldsymbol{i}} \boldsymbol{\varphi}-\boldsymbol{u}_{\boldsymbol{L} \boldsymbol{i}}\right) \boldsymbol{o}_{\boldsymbol{i}} \boldsymbol{o}_{\boldsymbol{i}} \boldsymbol{I}+ \\
& +m g \boldsymbol{g}_{\boldsymbol{S}}^{\boldsymbol{I}}
\end{aligned}
$$

the equation may be modified by

$$
\boldsymbol{\Omega}_{\boldsymbol{i}}=\left[\begin{array}{ccc}
o_{x i}^{2} & o_{x i} o_{y i} & o_{x i} o_{z i} \\
o_{x i} o_{y i} & o_{y i}^{2} & o_{y i} o_{z i} \\
o_{x i} o_{z i} & o_{y i} o_{z i} & o_{z i}^{2}
\end{array}\right]
$$

The position vector $\Delta \boldsymbol{u}_{i}$ equals to zero in the submitted system. Then the final equation of the partial derivative of the potential energy $U$ by the position vector $u_{L}$ is 


$$
\begin{aligned}
& \frac{\partial U}{\partial \boldsymbol{u}_{\boldsymbol{L}}}=\sum_{i=1}^{n}-k_{i} \boldsymbol{P}_{\boldsymbol{i}} \boldsymbol{\varphi} \boldsymbol{\Omega}_{\boldsymbol{i}}+m g \boldsymbol{I} \boldsymbol{g}_{\boldsymbol{S}}= \\
& =\sum_{i=1}^{n}-k_{i} \boldsymbol{\Omega}_{\boldsymbol{i}} \boldsymbol{P}_{\boldsymbol{i}} \boldsymbol{\varphi}+m g \boldsymbol{g}_{\boldsymbol{S}}
\end{aligned}
$$

The partial derivative of the potential energy $U$ by the angular position vector $\boldsymbol{\varphi}$ analogously is

$$
\begin{aligned}
& \frac{\partial U}{\partial \varphi}= \\
& =\sum_{i=1}^{n} k_{i}\left(\boldsymbol{u}_{L}-\boldsymbol{P}_{\boldsymbol{i}} \boldsymbol{\varphi}-\boldsymbol{u}_{L i}\right) \boldsymbol{o}_{\boldsymbol{i}} \boldsymbol{o}_{\boldsymbol{i}}\left(-\boldsymbol{P}_{\boldsymbol{i}}\right)- \\
& -m g \boldsymbol{g}_{\boldsymbol{S}} \boldsymbol{R}_{\boldsymbol{S}} \boldsymbol{I}= \\
& =\sum_{i=1}^{n} k_{i}\left(-\boldsymbol{P}_{\boldsymbol{i}} \boldsymbol{\varphi}\right) \boldsymbol{\Omega}_{\boldsymbol{i}}\left(-\boldsymbol{P}_{\boldsymbol{i}}\right)+m g \boldsymbol{I} \boldsymbol{R}_{\boldsymbol{S}} \boldsymbol{g}_{\boldsymbol{S}}= \\
& =\sum_{j=1}^{i=1}-b_{j} \boldsymbol{L}_{j} \boldsymbol{\varepsilon}_{j} \boldsymbol{L}_{\boldsymbol{j}} \boldsymbol{\varphi}^{\prime}+m g \boldsymbol{R}_{\boldsymbol{S}} \boldsymbol{g}_{\boldsymbol{S}}
\end{aligned}
$$

\subsection{Dissipative energy}

The dissipative energy is defined as

$$
D=\sum_{j=1}^{n} \frac{1}{2} b_{j}{\delta_{j}^{\prime}}^{2}
$$

wherein $b_{j}$ is the damping coefficient of $j$-th damping link and $\delta_{j}^{\prime}$ its deformation velocity. The deformation velocity $\delta_{j}^{\prime}$ can be expressed as the scalar multiplication of the relative velocity $\Delta \boldsymbol{u}_{j}^{\prime}$ of its endpoint against the start point and the unit vector $\boldsymbol{e}_{j}$ in the damping link direction.

The deformation velocity $\delta_{j}^{\prime}$ is then

$$
\delta_{j}^{\prime}=\Delta \boldsymbol{u}_{j}^{\prime} \boldsymbol{e}_{j}=\left(\boldsymbol{u}_{j}^{\prime}-\boldsymbol{u}_{L j}^{\prime}\right) \boldsymbol{e}_{j}
$$

The endpoint velocity $\boldsymbol{u}_{j}^{\prime}$ of $j$-th damping link is

$$
\boldsymbol{u}_{j}^{\prime}=\boldsymbol{u}_{L}^{\prime}+\varphi^{\prime} \times \boldsymbol{l}_{j}
$$

this equation, analogously to the equation (3) and (4), becomes

$$
u_{j}^{\prime}=u_{L}^{\prime}-L_{j} \varphi^{\prime}
$$

wherein $\boldsymbol{L}_{j}$ is antisymmetric tensor related to the vector $\boldsymbol{I}_{i}$, and is

$$
\boldsymbol{L}_{\boldsymbol{j}}=\left[\begin{array}{ccc}
0 & -l_{z j} & l_{y j} \\
l_{z j} & 0 & -l_{x j} \\
-l_{y j} & l_{x j} & 0
\end{array}\right]
$$

The partial derivative of the dissipative energy $D$ by the velocity vector $\boldsymbol{u}_{L}^{\prime}$ is

$$
\frac{\partial D}{\partial \boldsymbol{u}_{\boldsymbol{L}}^{\prime}}=\sum_{j=1}^{n} b_{j}\left(\boldsymbol{u}_{\boldsymbol{L}}^{\prime}-\boldsymbol{L}_{\boldsymbol{j}} \boldsymbol{\varphi}^{\prime}-\boldsymbol{u}_{\boldsymbol{L} \boldsymbol{j}}^{\prime}\right) \boldsymbol{e}_{\boldsymbol{j}} \boldsymbol{e}_{\boldsymbol{j}} \boldsymbol{I}
$$

The equation (30) may be modified by

$$
\varepsilon_{\boldsymbol{j}}=\left[\begin{array}{ccc}
e_{x j}^{2} & e_{x j} e_{y j} & e_{x j} e_{z j} \\
e_{x j} e_{y j} & e_{y j}^{2} & e_{y j} e_{z j} \\
e_{x j} e_{z j} & e_{y j} e_{z j} & e_{z j}^{2}
\end{array}\right]
$$

The velocity vector $\Delta u_{L}^{\prime}$ equals to zero in the submitted system. Then the final equation of the partial derivative of the dissipative energy $D$ by the velocity vector $\boldsymbol{u}_{L}^{\prime}$ is

$$
\frac{\partial D}{\partial \boldsymbol{u}_{\boldsymbol{L}}^{\prime}}=\sum_{j=1}^{n}-b_{j} \boldsymbol{L}_{\boldsymbol{j}} \boldsymbol{\varphi}^{\prime} \boldsymbol{\varepsilon}_{\boldsymbol{j}}=\sum_{j=1}^{n}-b_{j} \boldsymbol{\varepsilon}_{\boldsymbol{j}} \boldsymbol{L}_{\boldsymbol{j}} \boldsymbol{\varphi}^{\prime}
$$

The partial derivative of the dissipative energy $D$ by the angular velocity vector $\boldsymbol{\varphi}^{\prime}$ analogously is

$$
\begin{aligned}
& \frac{\partial D}{\partial \boldsymbol{\varphi}^{\prime}}= \\
& =\sum_{j=1}^{n} b_{j}\left(\boldsymbol{u}_{\boldsymbol{L}}^{\prime}-\boldsymbol{L}_{\boldsymbol{j}} \boldsymbol{\varphi}^{\prime}-\boldsymbol{u}_{\boldsymbol{L} \boldsymbol{j}}^{\prime}\right) \boldsymbol{e}_{\boldsymbol{j}} \boldsymbol{e}_{\boldsymbol{j}}\left(-\boldsymbol{L}_{\boldsymbol{j}}\right)= \\
& =\sum_{j=1}^{n} b_{j}\left(-\boldsymbol{L}_{\boldsymbol{j}} \boldsymbol{\varphi}^{\prime}\right) \boldsymbol{\varepsilon}_{\boldsymbol{j}}\left(-\boldsymbol{L}_{\boldsymbol{j}}\right)=\sum_{j=1}^{n}-b_{j} \boldsymbol{L}_{\boldsymbol{j}} \boldsymbol{\varepsilon}_{\boldsymbol{j}} \boldsymbol{L}_{\boldsymbol{j}} \boldsymbol{\varphi}^{\prime}
\end{aligned}
$$

\subsection{Differential equations of motion}

External forces $\boldsymbol{F}$ and external moments $\boldsymbol{M}$ are represented by generalized forces and moments in differential equations of motion.

Final differential equations of motion are

$$
\frac{d}{d t}\left(\frac{\partial K}{\partial \boldsymbol{u}_{L}^{\prime}}\right)_{x}+\left(\frac{\partial U}{\partial \boldsymbol{u}_{\boldsymbol{L}}}\right)_{x}+\left(\frac{\partial D}{\partial \boldsymbol{u}_{L}^{\prime}}\right)_{x}=F_{x}
$$

$$
\frac{d}{d t}\left(\frac{\partial K}{\partial \boldsymbol{u}_{L}^{\prime}}\right)_{y}+\left(\frac{\partial U}{\partial \boldsymbol{u}_{L}}\right)_{y}+\left(\frac{\partial D}{\partial \boldsymbol{u}_{L}^{\prime}}\right)_{y}=F_{y}
$$

$$
\frac{d}{d t}\left(\frac{\partial K}{\partial \boldsymbol{u}_{L}^{\prime}}\right)_{z}+\left(\frac{\partial U}{\partial \boldsymbol{u}_{L}}\right)_{z}+\left(\frac{\partial D}{\partial \boldsymbol{u}_{L}^{\prime}}\right)_{z}=F_{Z}
$$

$$
\frac{d}{d t}\left(\frac{\partial K}{\partial \boldsymbol{\varphi}^{\prime}}\right)_{x}+\left(\frac{\partial U}{\partial \boldsymbol{\varphi}}\right)_{x}+\left(\frac{\partial D}{\partial \boldsymbol{\varphi}^{\prime}}\right)_{x}=M_{x}
$$




$$
\begin{aligned}
& \frac{d}{d t}\left(\frac{\partial K}{\partial \varphi^{\prime}}\right)_{y}+\left(\frac{\partial U}{\partial \varphi}\right)_{y}+\left(\frac{\partial D}{\partial \varphi^{\prime}}\right)_{y}=M_{y} \\
& \frac{d}{d t}\left(\frac{\partial K}{\partial \varphi^{\prime}}\right)_{z}+\left(\frac{\partial U}{\partial \varphi}\right)_{z}+\left(\frac{\partial D}{\partial \varphi^{\prime}}\right)_{z}=M_{z}
\end{aligned}
$$

those are six differential equations that describe the spatial motion of the virtual body in the Cartesian coordinate system. Indexes mark the translational motion in the global coordinate system and mark the rotational motion around the axes of the global coordinate system.

\subsection{Numerical solution of differential equations}

The numerical solution of differential motion equations includes setting dynamical parameters of the solved system, kinematic excitation at the reference point of the virtual body and defining external forces and moments.

Positions $x, y$ and $z$ of the reference point $L$ are defined in differential equations (34) to (36), while external forces $F_{x}, F_{y}$ and $F_{z}$ are unknown variables.

External moments $M_{x}, M_{y}$ and $M_{z}$ equal to zero are defined in differential equations (37) to (39), while angular positions $\boldsymbol{\varphi}_{x_{1}}$ $\boldsymbol{\varphi}_{y}$ and $\boldsymbol{\varphi}_{z}$ around coordinate axes are unknown variables.

\subsection{Calculation of dynamical load}

The deformation $\delta_{i}$ of elastic links of linear guides may be calculated using the equation (15). The deformation velocity $\delta_{j}^{\prime}$ of damping links may be calculated using the equation (26).

The deformation $\delta_{i}$ multiplied by stiffness $k_{i}$ gives the result of the load, regarding the elastic links. The deformation velocity $\delta^{\prime}$ multiplied by damping coefficient $b_{j}$ gives the result of the load, regarding the damping links. The final dynamical load of a linear rolling guide is defined as a vector addition of the elastic and damping loads.

\section{PRACTICAL EXAMPLE}

The proposed calculation was verified on a chosen clamping frames manipulator that uses linear guides for the translational motion. This chapter briefly describes the design of the manipulator, its kinematic conditions and the numerical solution of the dynamical load with results discussion.

The manipulator (Fig. 4) uses clamping frames for fixing car bodies in a welding process.

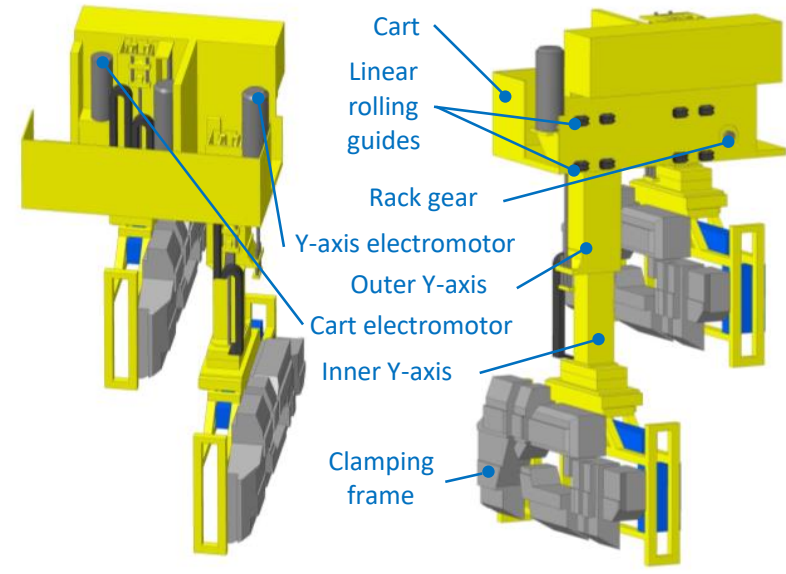

Figure 4. The clamping frames manipulator

The clamping frames are connected to the telescopic parts ( $\mathrm{Y}$ axis), those are included in the cart assembly. The cart assembly moves translationally on two parallel guiding profiles using eight linear guide carriages and is powered by the electromotor. The power from the electromotor is transmitted and transformed through the spur gear - through the meshing point of the pinion and the rack. The $\mathrm{Y}$-axis uses two telescopic parts, outer and inner ones; those enable the vertical motion of clamping frames.

\subsection{Numerical solution of the manipulator}

The motion of the manipulator may be understood as the motion of the absolute translational motion and the relative rotation around the reference point $L$. The $L$ point is also the mashing point of the pinion and the rack in the spur gear and the origin of the local coordinate system (Fig. 5).

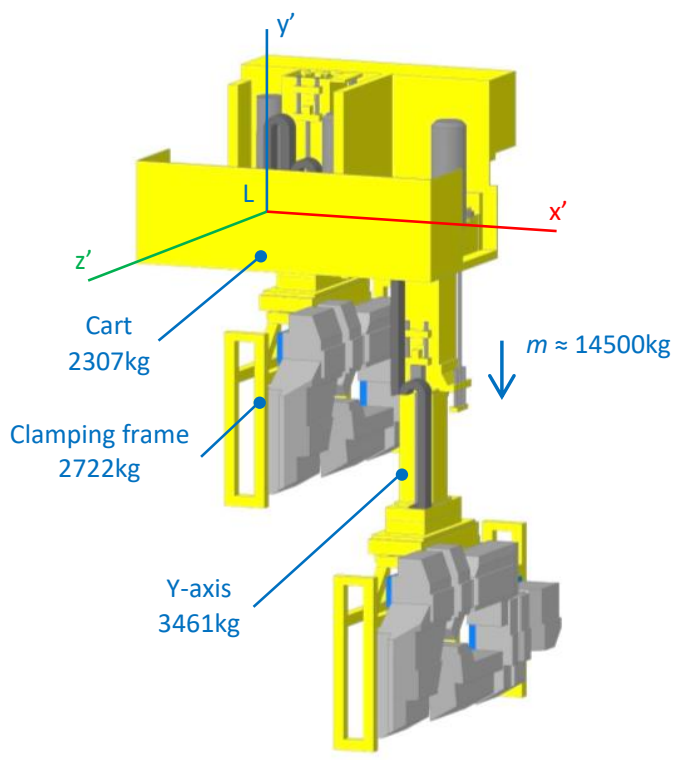

Figure 5. The clamping frames manipulator

The relative rotational motion is expressed as an oscillation around the mashing point of the spur gear. The relative rotational motion is expressed as an oscillation around the mashing point of the spur gear and is caused by the clearance and finite stiffness of linear guides and the whole system.

Mass and moments of inertia are concentrated into one rigid body. Elastic and damping links are shown in Fig. 7. Their values are initially obtained from a producer's documentation, where the stiffness in each direction is described. Nevertheless, the final values of stiffness and damping coefficient should also consider the flexibility of connected objects. These might be reached by experimental and numerical methods.

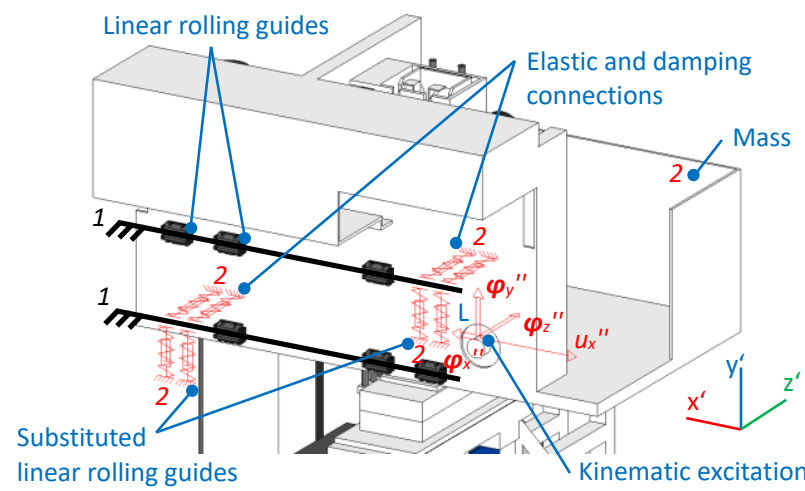

Figure 6. The kinematic scheme of the manipulator: 1 - Guiding profile, 2 - Mass

Only two linear guides are assumed for load transmission, the load is usually not distributed equally. In this case, two linear 
guides with the highest wear after several years in service are included in the calculation. For sufficient substitution of the linear rolling guide, four elastic and damping connections were used - two pairs at each end point of the linear carriage.

The cart electromotor is electronically controlled by a frequency converter and an incremental sensor of rotations $n_{M}$. Measured rotations $n_{M}$ that correspond to the cart translational motion under the maximal appearing load, are shown in Fig. 7.

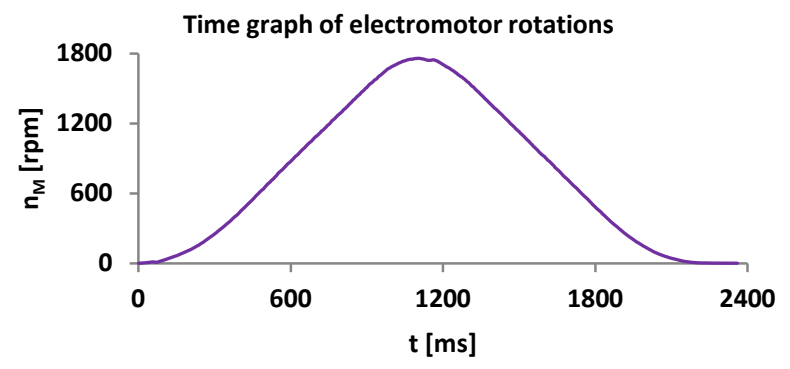

Figure 7. Time graph of electromotor rotations $n_{M}$

The cart velocity is obtained from the measured rotations and then the cart acceleration $a_{M}$ by a derivative of the velocity.

The solved system has four degrees of freedom, those are the translational motion in the $x$ axis direction and rotational motion around the $x, y$ and $z$ axes. The external moments equal to zero $\boldsymbol{M}_{\boldsymbol{x}}=0, \boldsymbol{M}_{\boldsymbol{y}}=0$ a $\boldsymbol{M}_{\mathbf{z}}=0$. The acceleration in the $\boldsymbol{x}$ axis direction $u_{x}{ }^{\prime \prime}$ is defined as the cart acceleration $a_{M}$. Accelerations in the $y$ and $z$ axis direction equal to zero $u_{y}{ }^{\prime \prime}=0$ and $u_{z}{ }^{\prime \prime}=0$.

It should be noted that for solving the system is necessary to find a static position first, and then initial conditions may be set.

\subsection{Dynamical load of linear rolling guides}

The dynamical load of the linear guide was calculated respecting the simulation of operating conditions of the representative manipulator. Time graphs Fig. 8 and Fig. 9 show the dynamical character of the linear guide 1 load in $y$ and $z$ axis direction.

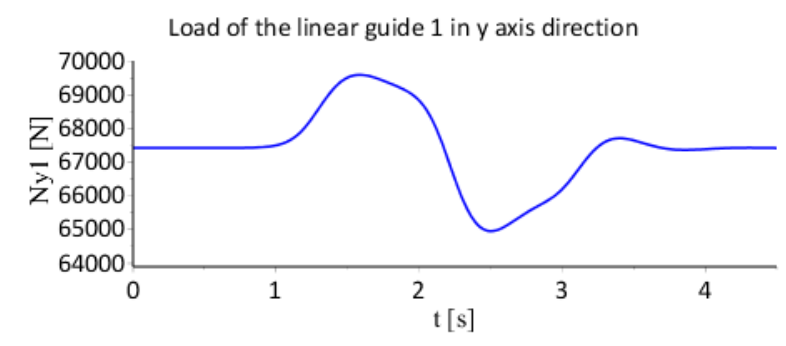

Figure 8. Load of the linear guide 1 in $y$ axis direction

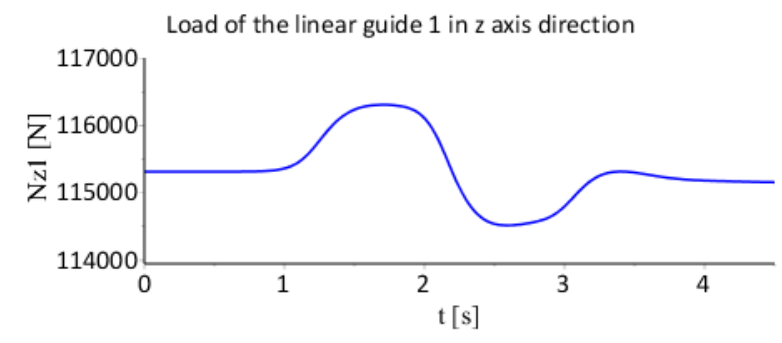

Figure 9. Load of the linear guide 2 in $z$ axis direction

Time graphs Fig. 10 and Fig. 11 show the dynamical character of the linear guide 2 load in $y$ and $z$ axis direction.

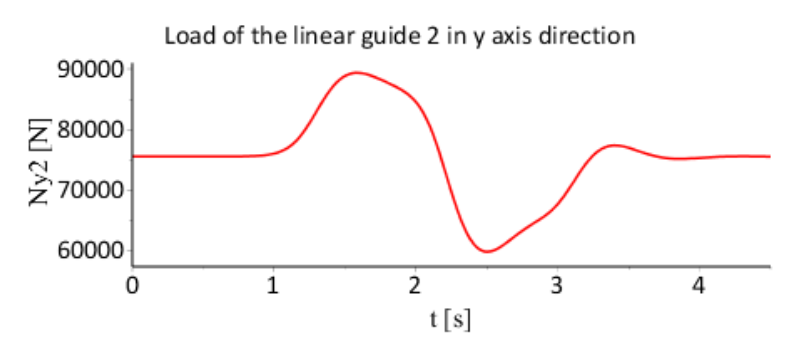

Figure 10. Load of the linear guide 2 in $y$ axis direction

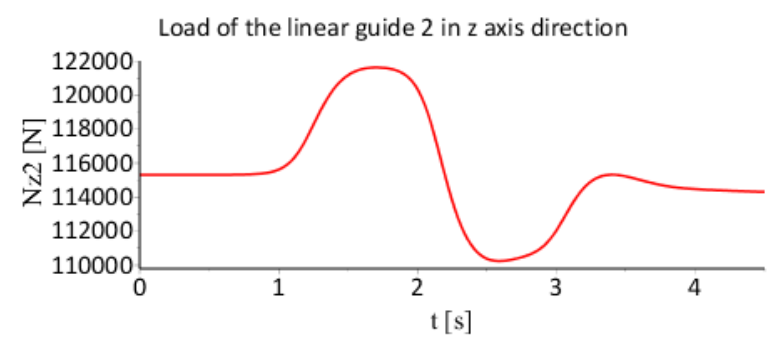

Figure 11. Load of the linear guide 2 in $z$ axis direction

The total dynamical load of linear guides is given by the vector addition in $y$ and $z$ direction.

The influence of the dynamical load is dependent on a wide range of conditions - such as mass parameters of connected bodies, kinematic conditions and on the position of linear guides in the system In this case, the dynamical load reached significant values.

\section{CONCLUSIONS}

The article showed a method of the dynamical load calculation to linear rolling guides, and verified this method on the representative manipulator. In practice, the method provides, clear and sufficient computations that are based on the mathematical model uses tensor calculus. Different dynamical parameters and kinematic conditions are easily set and controlled during all steps of the calculation.

The proposed method is applicable to a wide range of machines that use the linear rolling guides for translational motion. However, the accuracy of the calculation is dependent on values of acting dynamical forces given by structural mechanisms of the machine solved. These forces are not appropriately included. The accuracy of set dynamical parameters is a further limiting factor of proposed computation. The dynamical parameters set directly influence the result of dynamical load.

In the future, the method may be extended to a multibody system, thus giving a more accurate description of machines dynamical behaviour.

\section{ACKNOWLEDGMENTS}

This publication was written at the Technical University of Liberec as part of the project "Vibration damage identification of linear guiding system" with the support of the Specific University Research Grant, as provided by the Ministry of Education, Youth and Sports of the Czech Republic in the year 2020.

\section{REFERENCES}

[Cheng 2018] Cheng, D.J.et al. Improved friction model for the roller LM guide considering mechanics analysis. Journal of Mechanical Science and Echnology, 
January 2018, Vol. 32, No. 6, pp. 2723-2734. ISSN 1738-494X

[Feng 2018] Feng, H. et al. Feature extraction for fault diagnosis based on wavelet packet decomposition: An application on linear rolling guide. Advances in Mechanical Engineering, August 2018, Vol. 10, No. 8, ISSN 1687-8140

[HAGER Sondermaschinenbau 2020] HAGER Sondermaschinenbau. Available from $<$ https://www.hager-gmbh.de/grafik/titel-glas.jpg>

[Kwon 2019] Kwon, S.W. et al. Five-Degrees-of-Freedom Model for Static Analysis of Linear Roller Bearing Subjected to External Loading. Proceedings of the Institution of Mechanical Engineers, Part C-Journal of Mechanical Engineering Science, April 2019, Vol. 233, No. 8, pp. 2920-2938. ISSN 0954-4062

[Li 2018] Li, L. and Yang, J. A study of dynamic behaviors of contact pair in lubricated ball linear guide. Industrial Lubrication and Tribology, 2018, Vol. 70, No. 4, pp. 746-753. ISSN 0036-8792

[Ohta 2000] Ohta, H. and Hayashi, E. Vibration of Linear Guideway Type Recirculating Linear Ball Bearings. Journal of Sound and Vibration, August 2000, Vol. 235, No. 5, pp. 847-861. ISSN 0022-460X

[Ohta 2019] Ohta, H. et al. Reduction of Sticking in a LinearGuideway Type Recirculating Ball Bearing. Journal of Tribology-Transactions of the ASME, February 2019, Vol. 141, No. 2. ISSN 0742-4787

[Schaeffler Group 2020] Schaeffler Group. Available from < https://www.schaeffler.cz/remotemedien/media/_ shared_media_rwd/05_products_services_1/indust rial_products_services/linear_guidance_systems/00
01989C 16 9-schaeffler-produkterollenumlaufeinheiten-1_rwd_600.jpg >

[Schaeffler Technologies 2017] Schaeffler Technologies. Method for lubricating a linear guide, and linear guide. Publication WO 2018233750 A1, 21.6.2017

[Schiehlen 2007] Schiehlen, W. Trends in multibody system dynamics. Multibody system dynamics, May 2007, Vol. 18, No. 1. ISSN 1384-5640

[Shimizu 2008] Shimizu, S. et al. Study on the Life Distribution and Reliability of Roller-Based Linear Bearing. Tribology Transactions, 2008, Vol. 51, No. 4, pp. 446-453. ISSN 1040-2004

[Tao 2013] Tao, W. et al. Model for wear prediction of roller linear guides. Wear, 2013, Vol. 305, No. 1-2, pp. 260-266, ISSN 0043-1648

[THK 2011] THK. Condition-detecting device, method, and program, and information-recording medium. Granted Patent EP 1598569 B1, 31.8.2011

[THK 2019] THK. Method for diagnosing state of rolling guide device. Publication US 2019/0128330 A1, 2.5.2019

[Wei 2017a] Wei, W. et al. Dynamic reliability analysis of linear guides in positioning precision. Mechanism and Machine Theory, Vol. 116, pp. 451-464. ISSN 0094$114 \mathrm{X}$

[Wei 2017b] Wei, W. et al. Effects of wear on dynamic characteristics and stability of linear guides. Meccanica, Vol. 52, No. 11-12, pp. 2899-2913, ISSN 0025-6455

[Wittenburg 2008] Wittenburg, W. Dynamics of Multibody Systems. Springer-Verlag, 2007. ISBN 978-3-54073913-5

\section{CONTACTS}

prof. Ing. Lubomir Pesik, CSc.

Technical University of Liberec, Faculty of Mechanical Engineering Studentska 1402/2, Liberec, 461 17, Czech Republic +420 485353 312, lubomir.pesik@tul.cz, https://www.tul.cz/ 